\title{
Wave-front phase-modulation control and focusing of second-harmonic light generated in transparent nonlinear random structures
}

\author{
Can Yao, ${ }^{1}$ Francisco J. Rodriguez, ${ }^{1}$ Jorge Bravo-Abad, ${ }^{2}$ and Jordi Martorell ${ }^{1,3}$ \\ ${ }^{1}$ ICFO-Institut de Ciencies Fotoniques, Mediterranean Technology Park, 08860 Castelldefels, Barcelona, Spain \\ ${ }^{2}$ Departamento de Física Teórica de la Materia Condensada, Universidad Autónoma de Madrid, E-28049 Madrid, Spain \\ ${ }^{3}$ Departament de Física i Enginyeria Nuclear, Universitat Politècnica de Catalunya, Terrassa, Spain
}

(Received 10 August 2012; published 4 June 2013)

\begin{abstract}
We theoretically investigate how phase-only spatial light modulation can enable controlling and focusing the second-harmonic light generated in transparent nonlinear random structures. The studied structures are composed of domains with random sizes and antiparallel polarization, which accurately model widely used ferroelectric crystals such as strontium barium niobate. Using a first-principles Green-function formalism, we account for the effect that spatial light modulation of the fundamental beam introduces into the second-order nonlinear frequency conversion occurring in the considered class of structures. This approach provides a complete description of the physical origin of the second-harmonic light generation in the system, as well as the optimization of the light intensity in any arbitrary direction. Our numerical results show how the second-harmonic light is influenced by both the disorder in the structure and the boundaries of the crystal. Particularly, we find that the net result from the interplay between disorder and boundary effects is strongly dependent on the dimensions of the crystal and the observation direction. Remarkably, our calculations also show that although in general the maximum possible enhancement of the second-order light is the same as the one corresponding to linear light scattering in turbid media, in the Cerenkov phase matching direction the enhancement can exceed the linear limit. The theoretical analysis presented in this work expands the current understanding of light control in complex media and could contribute to the development of a new class of imaging and focusing techniques based on nonlinear frequency mixing in random optical materials.
\end{abstract}

DOI: 10.1103/PhysRevA.87.063804

PACS number(s): 42.65.Ky, 42.79.Hp, 42.30.Ms, 87.63.lm

\section{INTRODUCTION}

Recently, many efforts in the field of nonlinear optics have been made to understand the second-harmonic generation (SHG) phenomena occurring in random structures, both from theoretical and experimental standpoints [1-7]. Nowadays, it is widely accepted that the SHG from disordered media grows linearly with the length of the random media along the fundamental beam path [1,2]. On the other hand, it is also well known that a SHG speckle pattern is formed even in materials that are transparent, but characterized by nonlinear random structures [4]. An example of this class of structures is the strontium barium niobate (SBN) crystal, which is composed of domains with random sizes and antiparallel polarization [8-12]. The SHG speckle obtained in this crystal spans a wide range of emission directions, which greatly reduces its efficiency in one particular direction. As it has been experimentally demonstrated, a speckle-free generation can be recovered by a wave-front phase-modulation method to focus the SHG from a SBN crystal from many speckles to a single point [13]. That approach is based on a concept similar to the one used to focus the fundamental beam through strongly scattering turbid media [14-23].

In this paper, we present a detailed theoretical study of the spatial intensity control of SHG by fundamental beam phase modulation. A random nonlinear structure is considered, with random domain size and antiparallel polarization. In our analysis, we first compute the SHG from one single domain by means of the Green-function formalism. Then, the SHG from the whole structure is obtained as the coherent sum of SHG from each of the domains forming the system.
Our calculations show that, in some particular directions, the presence of the crystal boundary can play a role as important as the disorder of the structure in determining the behavior and optimal enhancement efficiency of the SHG. In any other generic direction different from those special directions, the SHG is mainly contributed from the disordered structure, and the boundary effect is negligible. It is also shown that the maximum SHG enhancement obtained by considering just the disordered structure contribution is comparable with the enhancement efficiency obtained in light focusing through linear diffusive media. However, in some of the abovementioned special directions (specifically in Cerenkov phase matching directions), the effect of the crystal boundary can be significant enough to lead to enhancement efficiencies larger than those reached in linear diffusive media.

This paper is organized as follows. In Sec. II we present the theoretical framework for the second harmonic intensity (SHI) generated from a nonlinear random crystal composed of domains with random sizes and opposite polarizations. The SHI from one single domain is obtained by solving the secondorder wave equation by means of a Green function. Then, the SHI from the whole structure is deduced as the coherent sum of SH light from all the domains. The optimization of the SHI in a certain direction is realized by introducing an extra phase term to the plane-wave fundamental light that depends on the transversal spatial coordinates. In Sec. III we first discuss the SHG from a homogeneous single crystal as a function of the crystal size and the observation direction. Then, we carry out the same analysis for the case of a random structure. Both the SHI under plane-wave incidence and the optimized SHI by wave-front phase modulation are analyzed. We discuss 
the results comparing them with the case of the optimization of light transmission in diffusive linear media.

\section{THEORETICAL FRAMEWORK}

We consider a crystal structure characterized by a homogeneous refractive index but featuring random changes in the second-order nonlinear susceptibility. More specifically, we focus our discussion in a structure similar to the one that can be found in ferroelectric crystals such as $\mathrm{SBN}$ or $\mathrm{LiNbO}_{3}$. In such crystals, long domains are formed along the crystallographic $c$ axis with antiparallel orientation. In the case of SBN, the size and position of the domains are usually randomly distributed. A random distribution can also be introduced in $\mathrm{LiNbO}_{3}$ by electric-field poling with designed patterns. The elongated shape of the domains implies that the nonlinear light emission from a fundamental beam propagating perpendicular to the $c$ axis is mostly confined to the plane perpendicular to the $c$ axis $[3,6]$.

The wave equation governing the SH electric field $E^{(2 \omega)}$, as directly deduced from Maxwell equations for nonmagnetic, nonconductive materials, can be written as

$$
\nabla \times \nabla \times \mathbf{E}^{(\mathbf{2} \omega)}-k^{(2 \omega) 2} \mathbf{E}^{(\mathbf{2} \omega)}=\frac{(2 \omega)^{2}}{c^{2}} \mathbf{P}^{(\mathbf{2} \omega)},
$$

where $\mathbf{P}^{(2 \omega)}$ is the component of the material polarization oscillating at the SH frequency, and $k^{(2 \omega)}=(2 \omega / c) n^{(2 \omega)}$ is the wave number of the $\mathrm{SH}$ wave. The Green-function formalism can be used to solve this equation. Such formalism has been widely used in electromagnetism [24,25] and optics [26], including for problems in nonlinear optics [3,27-29]. When a homogeneous refractive index is assumed, the electric field at position $\boldsymbol{r}$ can be represented by the integral over the nonlinear volume,

$$
\mathbf{E}^{(\mathbf{2} \omega)}(\boldsymbol{r})=\frac{(2 \omega)^{2}}{c^{2}} \int_{V} \stackrel{\leftrightarrow}{G}\left(\boldsymbol{r}, \boldsymbol{r}^{\prime}\right) \mathbf{P}^{(2 \omega)}\left(\boldsymbol{r}^{\prime}\right) d \boldsymbol{r}^{\prime},
$$

where the dyadic Green function is given by

$$
\stackrel{\leftrightarrow}{G}\left(\boldsymbol{r}, \boldsymbol{r}^{\prime}\right)=\left(\stackrel{\leftrightarrow}{1}-\frac{\nabla \nabla}{k^{(2 \omega) 2}}\right) \frac{e^{i k^{(2 \omega)}\left|\boldsymbol{r}-\boldsymbol{r}^{\prime}\right|}}{4 \pi\left|\boldsymbol{r}-\boldsymbol{r}^{\prime}\right|} .
$$

We are interested in the far-field distribution of the SH light. Thus, we take the asymptotic expansion of $\overleftrightarrow{G}\left(\boldsymbol{r}, \boldsymbol{r}^{\prime}\right)$ in the limit $r \gg r^{\prime}$. We make the approximation $\left|\boldsymbol{r}-\boldsymbol{r}^{\prime}\right| \approx r-\hat{\mathbf{r}} \cdot \mathbf{r}^{\prime}$ and keep only the terms in $1 / r$. The result can be represented as

$$
\stackrel{\leftrightarrow}{G}\left(\boldsymbol{r}, \boldsymbol{r}^{\prime}\right)=\left(\stackrel{\leftrightarrow}{1}-\hat{\mathbf{k}}^{(\mathbf{2} \omega)} \otimes \hat{\mathbf{k}}^{(\mathbf{2} \omega)}\right) \frac{e^{i k^{(2 \omega)}\left(r-\hat{\mathbf{r}} \cdot \mathbf{r}^{\prime}\right)}}{4 \pi r} .
$$

In this equation, $\hat{\mathbf{k}}^{(\mathbf{2} \omega)}$ corresponds to a unitary vector in the direction of observation of the $\mathrm{SH}$ field and $\otimes$ is the tensor product. Finally, taking the material polarization at the SH frequency as $\mathbf{P}^{(2 \omega)}\left(\mathbf{r}^{\prime}\right)=\overleftrightarrow{\chi}^{(2)}\left(\mathbf{r}^{\prime}\right): \mathbf{E}^{(\omega)}\left(\mathbf{r}^{\prime}\right) \mathbf{E}^{(\omega)}\left(\mathbf{r}^{\prime}\right)$ and the fundamental field as $\mathbf{E}^{(\omega)}(\mathbf{r})=\mathbf{E}^{(\omega)} e^{i \mathbf{k}^{(\omega)} \cdot \mathbf{r}}$ we can write in Cartesian components,

$$
\begin{aligned}
E_{i}^{(2 \omega)}= & \frac{(2 \omega)^{2} e^{i k^{(2 \omega)} r}}{c^{2} 4 \pi r} \int_{V}\left(\delta_{i j}-\hat{k}_{i}^{(2 \omega)} \hat{k}_{j}^{(2 \omega)}\right) \\
& \times\left(\chi_{j k l}^{(2)} E_{k}^{(\omega)} E_{l}^{(\omega)}\right) e^{i \Delta \mathbf{k} \cdot \mathbf{r}^{\prime}} d \boldsymbol{r}^{\prime},
\end{aligned}
$$

where $\Delta \mathbf{k}=\mathbf{k}^{(2 \omega)}-2 \mathbf{k}^{(\omega)}$ is the wave-vector mismatch. If we assume that $\stackrel{\leftrightarrow}{\chi}^{(2)}$ is constant over the nonlinear volume, as it will happen for a single crystalline domain, it is possible to take it out of the integral. In such case, the electric field will be proportional to $\int_{V} e^{i \Delta \mathbf{k} \cdot \mathbf{r}^{\prime}} d \boldsymbol{r}^{\prime}$, and this integral contains all the angular dependence of the $\mathrm{SH}$ emission. The integral has an analytic solution for some simple volume shapes [3].

For the case of long domains, such as those found in SBN, irradiated by a fundamental wave that propagates normal to the long axis (crystal $c$ axis), the light emission is confined mostly to the plane normal to the long axis. This can be deduced from Eq. (5) and is also observed experimentally $[3,6]$. Without loss of generality, and in order to simplify the theoretical discussion, we restrict our analysis to the two-dimensional (2D) plane perpendicular to the $c$ axis. Since both fundamental and SHG light propagate along that plane, no fundamental difference is expected for a full three-dimensional (3D) treatment. We describe the plane with $x-y$ Cartesian coordinates and take $x$ as the fundamental beam propagation direction (Fig. 1). The integral in Eq. (5) has analytical solution for a rectangular-shaped domain with dimensions $L_{x}$ and $L_{y}$ along the $x$ and $y$ directions, respectively [3]. If the domain is located at position $(x, y)$ we can write

$$
\begin{aligned}
E_{(x, y)}^{(2 \omega)}= & \frac{2 \omega^{2} e^{i 2 \omega n_{2 \omega} r / c}}{c^{2} \pi r} d_{\mathrm{eff}} E^{(\omega)} E^{(\omega)} e^{i\left(x \Delta k_{x}+y \Delta k_{y}\right)} L_{x} L_{y} \\
& \times \operatorname{sinc}\left(\Delta k_{x} L_{x} / 2\right) \operatorname{sinc}\left(\Delta k_{y} L_{y} / 2\right),
\end{aligned}
$$

where $d_{\text {eff }}$ is the effective component of the second-order nonlinear susceptibility and $r$ is the distance to the observation point. The phase mismatch between the fundamental and SHG light in the $x$ and $y$ directions are $\Delta k_{x}=4 \pi\left(n_{2 \omega} \cos \phi-\right.$ $\left.n_{\omega} \cos \phi_{i n}\right) / \lambda_{\omega}$ and $\Delta k_{y}=4 \pi\left(n_{2 \omega} \sin \phi-n_{\omega} \sin \phi_{i n}\right) / \lambda_{\omega}$ respectively. $n_{\omega}$ and $n_{2 \omega}$ are the refractive indices of fundamental and $\mathrm{SH}$ light, $\lambda_{\omega}$ is the fundamental wavelength, and $\phi$ and $\phi_{i n}$ are the SHG output angle and fundamental incident angle to the forward direction, respectively. Without loss of generality, we assume that the forward direction corresponds to the fundamental incident direction, thus $\phi_{i n}=0$.
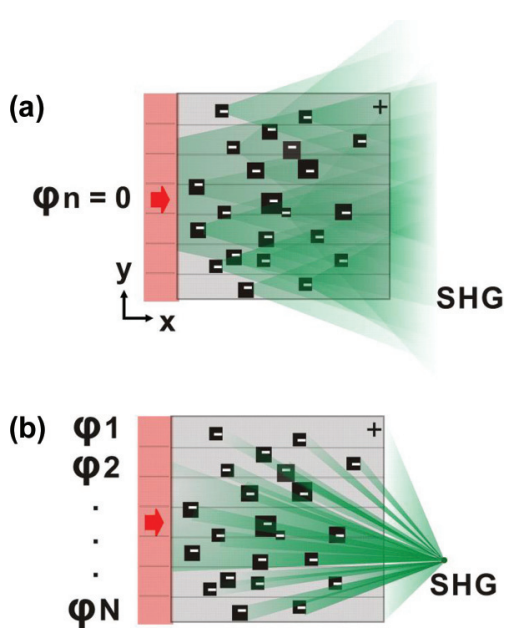

FIG. 1. (Color online) Schematic of SHG from a nonlinear structure with random domain size and antiparallel polarization: (a) SHG under plane-wave incidence. (b) SHG optimized at one point by wave-front modulation. 
Once the SH electric field from a single domain has been calculated, the SH electric field from the whole structure can be obtained as the result of the interference of the SHG from each domain. This can be understood as splitting the integral in Eq. (5) in a sum over all the nonlinear domains ( $n$ ) within which the nonlinear susceptibility is constant:

$$
\begin{aligned}
E^{(2 \omega)} & =\frac{2 \omega^{2} e^{i 2 \omega n_{2 \omega} r / c}}{c^{2} \pi r} E^{\omega} E^{\omega} \sum_{n=1}^{N} d_{\mathrm{eff}}(n) \int_{V(n)} e^{i \Delta \mathbf{k} \cdot \mathbf{r}^{\prime}} d \boldsymbol{r}^{\prime} \\
& =\sum_{n=1}^{N} E_{n}^{(2 \omega)} .
\end{aligned}
$$

Note that, although Eq. (7) is already written in the scalar form, the same would be valid in the general 3D treatment in vector form.

Next we introduce a phase modulation of the fundamental beam along the perpendicular direction $(y)$. Specifically, the beam is divided into $N$ segments, and the phase of each segment $\varphi_{n}(1 \leqslant n \leqslant N)$ is modulated independently between 0 and $2 \pi$. If we assume that the fundamental beam is perfectly collimated, and by neglecting the effect of beam diffraction during propagation within the crystal, the $\mathrm{SH}$ signal generated by the mix of the fundamental beam from two different phase segments can be neglected. This allows increasing the computational efficiency of our theoretical treatment, as we need to consider only the SH generated from each segment separately.

In this calculation we found it convenient to divide the structure into $N$ groups corresponding to the segments whose phases are modulated. Thus, if we assume that there are $M$ domains in each of the $N$ groups modulated by phases $\varphi_{n}$, we can write

$$
E^{2 \omega}=\sum_{n=1}^{N} \sum_{m=1}^{M} E_{x_{m n}, y_{m n}}^{2 \omega} e^{i 2 \varphi_{n}}
$$

In the crystal structure considered, the absolute value of the effective nonlinear susceptibility is constant and only its sign changes for domains with opposite polarization. If we take $P_{m n}$ with value 1 or -1 as the sign given by the polarization of domain $m n$, from Eqs. (6) and (8), the SHI can be expressed as

$$
\begin{aligned}
I^{2 \omega}= & d \mid \sum_{n=1}^{N} \sum_{m=1}^{M} P_{m n} L_{x_{m n}} L_{y_{m n}} e^{i\left(x_{m n} \Delta k_{x}+y_{m n} \Delta k_{y}\right)} \\
& \times\left.\operatorname{sinc}\left(\frac{\Delta k_{x} L_{\mathrm{x}_{m n}}}{2}\right) \operatorname{sinc}\left(\frac{\Delta k_{y} L_{\mathrm{y}_{m n}}}{2}\right) e^{i 2 \varphi_{n}}\right|^{2},
\end{aligned}
$$

where we denote $d=8 \omega^{4} n_{2 \omega} d_{\mathrm{eff}}^{2} I^{\omega} I^{\omega} / c^{5} \pi^{2} r^{2} n_{\omega}^{2} \varepsilon_{0}$. As deduced from Eq. (9), the maximum SHI occurs when the modulated phases $\varphi_{n}$ compensate the phases introduced by the rest of the terms. With the appropriate values for $\varphi_{n}$, the contributions from the $N$ modulated groups can be added in phase. We define the phase modulation SHG enhancement efficiency $\eta$
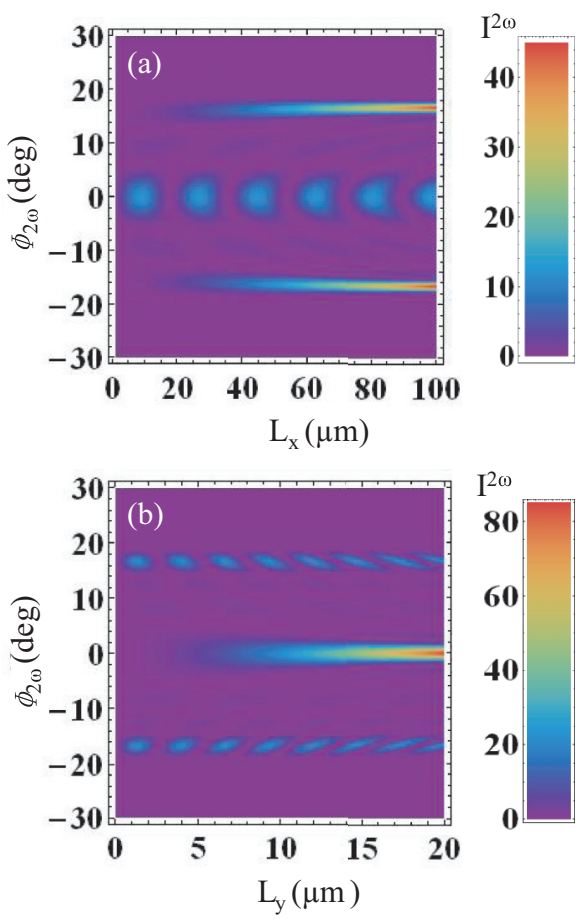

FIG. 2. (Color online) Spatial distribution of the SHI (in arbitrary units) from plane-wave input on a homogeneous crystal of $2.1 \mu \mathrm{m}$ width, and variable crystal length (a), and a homogeneous crystal of $18 \mu \mathrm{m}$ length, and variable crystal width (b).

as the proportion between the maximum $I^{2 \omega}$ and the $I^{2 \omega}$ under plane-wave input, $\eta=I^{2 \omega}\left(\varphi_{n}\right)_{\max } / I^{2 \omega}\left(\varphi_{n}=0\right){ }^{1}$

\section{RESULTS}

\section{A. Homogeneous crystal}

We first consider the SHG from a homogeneous crystal. The expression for the SHI can be deduced from Eq. (6) as

$$
I^{2 \omega}=d L_{x}^{2} L_{y}^{2} \operatorname{sinc}^{2}\left(\frac{\Delta k_{x} L_{x}}{2}\right) \operatorname{sinc}^{2}\left(\frac{\Delta k_{y} L_{y}}{2}\right),
$$

where $L_{x}$ and $L_{y}$ are now the length and width of the whole crystal, respectively. In the case in which $\Delta k_{x}=0$ but $\Delta k_{y} \neq$ 0, Eq. (10) can be rewritten as

$$
I^{2 \omega}=\frac{4 d L_{x}^{2}}{\Delta k_{y}^{2}} \sin ^{2}\left(\frac{\Delta k_{y} L_{y}}{2}\right) .
$$

This situation will occur in usual experiments with SBN for an emission (internal) angle $\phi=16.8^{\circ}$. To calculate this angle, we have assumed the fundamental beam at wavelength $1064 \mathrm{~nm}$, and the SHG at $532 \mathrm{~nm}$. We then take the corresponding refractive index of the $\mathrm{Sr}_{0.61} \mathrm{Ba}_{0.39} \mathrm{Nb}_{2} \mathrm{O}_{6}$ crystal: $n_{1064 \mathrm{~nm}}=$ 2.22 and $n_{532 \mathrm{~nm}}=2.32$ [30]. From Eq. (11) it is evident that the SHI will have a quadratic growth with the crystal length $L_{x}$, and it will fluctuate periodically with $L_{y}$ (Fig. 2). This

\footnotetext{
${ }^{1}$ We applied the software MATHEMATICA using the Nmaximize function with the DifferentialEvolution method to obtain the maximum SHG enhancement efficiency.
} 
condition is also called Cerenkov phase matching [31-34]. In the same way, for the case of collinear SHG $\left(\phi=0^{\circ}\right)$, $\Delta k_{y}=0$ and $\Delta k_{x} \neq 0$. The intensity will have an expression like Eq. (11) but with a permutation of the subscripts $x$ and $y$. The SHI will follow a quadratic growth with the crystal width $L_{y}$, and it will show the well-known periodical oscillations with the crystal length $L_{x}$ (Fig. 2). Note that in this case, together with the growth of the crystal width, there is a linear growth of the fundamental wave intensity that propagates inside the crystal.

Both the collinear and the Cerenkov effects are due to the boundaries of the crystal and will persist even after we introduce randomness to the structure. For SHI at any angle $\phi$, for which neither $\Delta k_{x}$ nor $\Delta k_{y}$ is 0 , Eq. (10) can be simplified into

$$
I^{2 \omega}=\frac{16 d}{\Delta k_{x}^{2} \Delta k_{y}^{2}} \sin ^{2}\left(\frac{\Delta k_{x} L_{x}}{2}\right) \sin ^{2}\left(\frac{\Delta k_{y} L_{y}}{2}\right),
$$

which can be rather weak compared with the quadratic growth in the collinear and Cerenkov cases. In general, when we introduce randomness in the structure, the boundary effect will be negligible in these directions.

Now, we analyze the case in which the SHG from a homogeneous crystal is maximized for a certain angle. In this case, the input beam is divided into $N$ segments of width $L$ whose phases are modulated by $\varphi_{n}(1 \leqslant n \leqslant N)$ :

$$
\begin{aligned}
I^{2 \omega}= & d\left[L_{x} L \operatorname{sinc}\left(\frac{\Delta k_{x} L_{x}}{2}\right) \operatorname{sinc}\left(\frac{\Delta k_{y} L}{2}\right)\right]^{2} \\
& \times\left|\sum_{n=1}^{N} e^{i n L \Delta k_{y}} e^{i 2 \varphi_{n}}\right|^{2} .
\end{aligned}
$$

As deduced from Eq. (13), if we introduce a phase modulation in such a way that $\varphi_{n}$ compensates the phase from $n L \Delta k_{y}$, the maximized $I^{2 \omega}$ is proportional to $N^{2} L^{2}$. Therefore, $I^{2 \omega}$ features a quadratic growth with the crystal width $L_{y}=N L$, while it has the same relation with the crystal length $L_{x}$ as the $I^{2 \omega}$ under the plane-wave input.

The SHI under plane-wave input in Eq. (13) is obtained when $\varphi_{n}=0$ for all $n$. Then, the SHG enhancement efficiency is $\eta=N^{2} /\left|\sum_{n=1}^{N} e^{i n L \Delta k_{y}}\right|^{2}$. For the collinear case, $\eta\left(\phi=0^{\circ}\right)=$ 1 , and therefore $I^{2 \omega}$ cannot be optimized through this phase modulation method. For other angles, the precise value of the enhancement depends on the size of the modulated segments $L$. If $L=2 \pi / \Delta k_{y}$, the denominator in the expression for $\eta$ is $N^{2}$ and thus $\eta=1$ (no enhancement). In contrast, if the wave-front modulation is performed using small segments $\left(L \ll \pi / \Delta k_{y}\right)$ the denominator in $\eta$ can be approximated by $\left|\int_{0}^{N} e^{i n} d n\right|^{2}=2-2 \cos N$. This term corresponds to the effect of the crystal width (in this case proportional to $N$ ) in the intensity under plane-wave irradiation. The oscillation in the denominator is translated into an oscillation in $\eta$. The minima in the oscillation correspond to $\eta=N^{2} / 4$, that features a quadratic growth with $N$. Note also that the possible zeros in the denominator cause $\eta$ to diverge to infinity for certain values of $N$. However, this divergence, that has its origin in zero intensity under the plane wave for the corresponding crystal width, does not lead to an infinite value in the maximized intensity.

\section{B. Random structures}

A SHG speckle appears when some degree of randomness is introduced into the distribution of polarization of the domains, even if the domain size is uniform. The degree of disorder is quantified by the parameter $\sigma(0 \leqslant \sigma \leqslant 1)$, which measures the fraction of domains for which $P=1$ over the total number of domains. Figure 3 shows the SHG speckle pattern from a random structure, due to the interference of SHG from all domains, which are of uniform size $L$ and antiparallel polarization. For a random structure whose width is fixed, as the structure length increases, the speckle size decreases (except in $\phi=0^{\circ}$ ), whereas the speckle number increases. This behavior is associated with the fact that the speckle size is inversely proportional to the angular range from which the light arrives to the observation point [35]. By comparison of Figs. 3(a) and 3(b), one can see that if the whole structure size is fixed, as the domain size $L$ increases, the SHG becomes stronger in the forward direction.
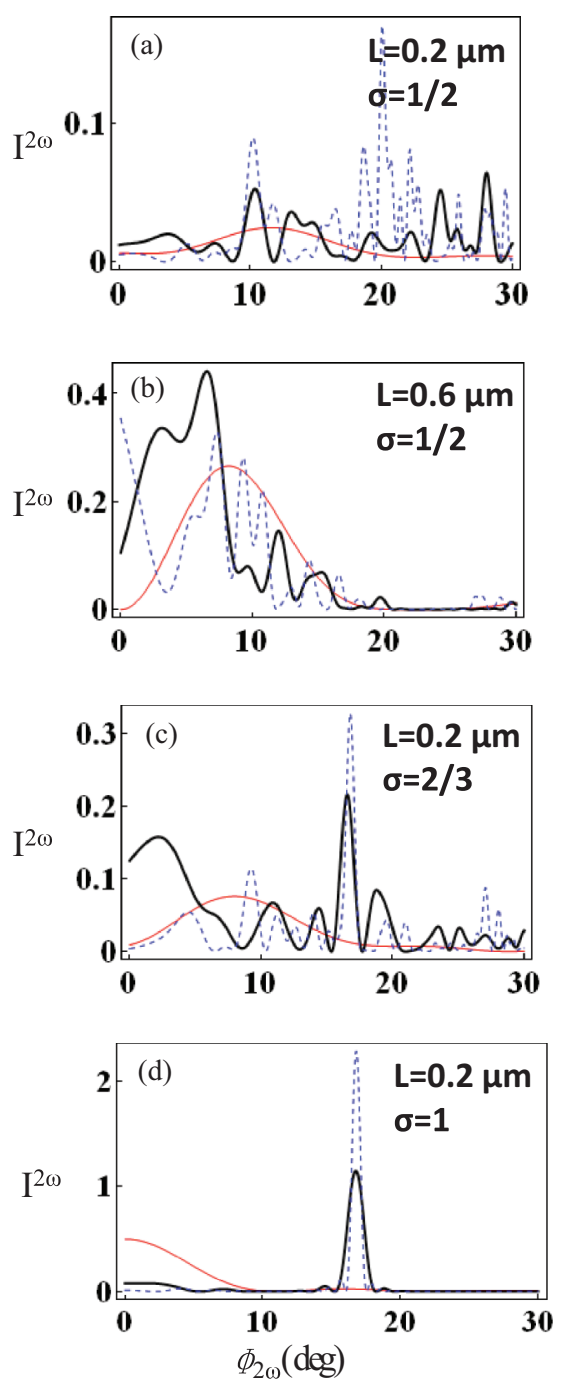

FIG. 3. (Color online) The intensity (in arbitrary units) of the SHG speckle pattern generated from plane-wave input on structures with uniform square domain size $L$ and random polarization. The crystal width is $1.2 \mu \mathrm{m}$, and the crystal length takes the following values: $0.6 \mu \mathrm{m}$ red, $30 \mu \mathrm{m}$ thick black, and $60 \mu \mathrm{m}$ dashed blue. 
If we gradually increase the randomness of the structure by decreasing $\sigma$ from 1 (homogeneous crystal) to $1 / 2$ (half of the domains with a given polarization), we find that, as the structure becomes more disordered, the Cerenkov SHG peak at angle $\phi=16.8^{\circ}$ due to the crystal boundary effect gradually disappears [Figs. 3(d), 3(c) and 3(a)]. The disorder effect in the crystal washes out the effect of the homogeneous crystal boundaries.

Now, we further introduce random sizes to the domains, together with the antiparallel polarization in the structure. In this case, the modeled structure consists of a group of domains with random sizes and negative polarization into a homogeneous crystal with positive polarization (Fig. 1). We assume the domains are square shaped, that is, $L_{x m n}=L_{y m n} \equiv$ $L_{m n}$, where $L_{m n}$ takes random values from $L_{\min }$ to $L_{\max }$. $L_{\max }$ corresponds also to the width of each phase segment along the $y$ direction, so that the domain position $x_{m, n}$ will randomly take values between $L_{m n}$ and $L_{x}$, and $y_{m, n}$ between $(n-1) L_{\max }+L_{m n}$ and $n L_{\max }$. In our simulations the eventual spatial overlap of two domains was not prevented. However, comparing the results with those obtained in simulations of constant sized domains for which there is no overlap, we conclude that the eventual overlap of domains does not affect the main conclusions presented here.

In this case, $\sigma$ corresponds to the proportion of the area covered by domains with polarization pointing up $(P=1)$ to the total structure area. From Eq. (9), we computed the SHG before and after optimization for three representative directions, namely, in the collinear angle, the Cerenkov angle, and an angle at which neither $\Delta k_{x}$ nor $\Delta k_{y}$ is 0 (Fig. 4).

If we now define

$$
\begin{aligned}
t_{n}= & \sum_{m=1}^{M} P_{m n} L_{m n}^{2} \operatorname{sinc}\left(\frac{\Delta k_{x} L_{m n}}{2}\right) \\
& \times \operatorname{sinc}\left(\frac{\Delta k_{y} L_{m n}}{2}\right) e^{i\left(x_{m n} \Delta k_{x}+y_{m n} \Delta k_{y}\right)},
\end{aligned}
$$

the maximum SHI enhancement by phase modulation can be expressed as

$$
\eta=\frac{I_{\max }^{2 \omega}}{I_{0}^{2 \omega}}=\frac{\left|\sum_{n=1}^{N} t_{n} e^{i 2 \varphi_{n}}\right|^{2}}{\left|\sum_{n=1}^{N} t_{n}\right|^{2}}=\frac{\left(\sum_{n=1}^{N}\left|t_{n}\right|\right)^{2}}{\left|\sum_{n=1}^{N} t_{n}\right|^{2}} .
$$

Now, we consider the average values $\langle\eta\rangle$ over a statistically significant number of structures with randomly distributed domain sizes, polarizations, and positions. In general, the phase of $t_{n}$ takes any value between 0 and $2 \pi$ with equal probability. Consequently, the average maximum SHI enhancement by phase modulation is $\eta=1+\pi(N-1) / 4$ (Fig. 5). Note that this expression for $\eta$ is in fact equal to the phase modulation enhancement through turbid linear media [36]. However, for the SHG in the Cerenkov and the collinear angle, the contribution from the structure boundaries $\left(I_{\text {bulk }}\right)$ that behaves like in the homogeneous crystal can be relatively large compared with the contribution from the random structure ( $\left.I_{\text {random }}\right)$.

In the collinear angle, $I_{\text {bulk }}$ is proportional to $L_{v}^{2}$, whereas $I_{\text {random }}$ is proportional to $L_{y}$. Thus, for a fixed crystal length $L_{x}$, $I_{\text {bulk }}$ plays a more important role as $L_{y}$ grows larger. For any random structure, the average enhancement in the collinear
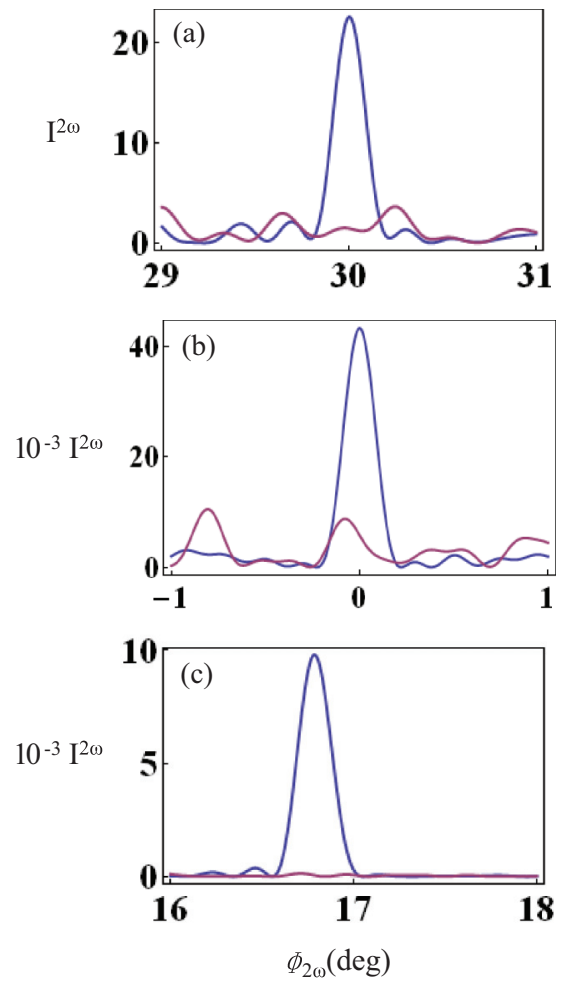

FIG. 4. (Color online) The SHI (in arbitrary units) as a function of the angle before and after optimization around $\phi=30^{\circ}$ (a), $\phi=0^{\circ}(\mathrm{b})$, and $\phi=16.8^{\circ}$ (c). The structure background consists of a homogeneous medium, $60 \mu \mathrm{m}$ wide and $30 \mu \mathrm{m}$ long, with positive polarization. Part of this medium is covered by domains with random sizes between 0 and $3 \mu \mathrm{m}$ and negative polarization. The phase segment number is $N=20$. The red line shows the SHI under plane-wave input, whereas the blue line corresponds to the SHI optimized by phase modulation.

angle can take values between 1 , due to the bulk boundary effect, and $1+\pi(N-1) / 4$, due to the disorder effect (Fig. 5).

In the Cerenkov angle, $I_{\text {bulk }}$ is proportional to $L_{x}^{2}$ whereas $I_{\text {random }}$ is proportional to $L_{x}$. For a fixed crystal width $L_{y}, I_{\text {bulk }}$ plays a more important role as $L_{x}$ grows larger. In general, the average enhancement in the Cerenkov angle could take values between $1+\pi(N-1) / 4$, due to the disorder effect, and $N^{2} /\left|\sum_{n=1}^{N} e^{i n L \Delta k_{y}}\right|^{2}$ due to the bulk boundary effect. If the phase modulated segments are chosen with the appropriate width, the effect of the crystal boundaries makes the average enhancement in the Cerenkov angle substantially larger than at other angles [Fig. 5(b)]. Note also in the example given in Fig. 5(a) the oscillation in $\langle\eta\rangle$ produced by a segment width $L<\pi / \Delta k_{y}$ as explained in Sec. III A.

From the analysis above it follows that, in either the collinear or Cerenkov direction, a larger crystal width results in a smaller $\eta$, closer to the minimum value in their individual efficiency ranges. This is due to the boundary effect in the collinear direction and to the disorder effect in the Cerenkov direction, respectively. On the other hand, a larger crystal length results in a larger $\eta$, closer to the maximum value in their individual efficiency ranges, in that case due to the disorder 

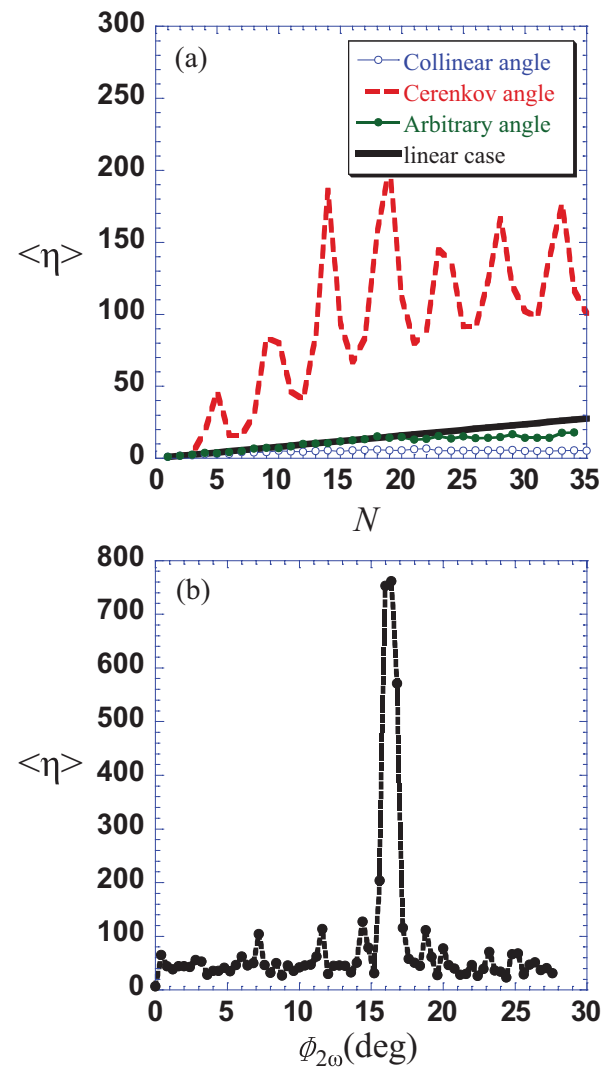

FIG. 5. (Color online) (a) Maximum SHI enhancement efficiency as a function of the phase segment number $N$, at an arbitrary angle $\phi=30^{\circ}$, collinear angle $\phi=0^{\circ}$, and Cerenkov angle $\phi=16.8^{\circ}$. The black solid line corresponds to the linear enhancement efficiency $1+\pi(N-1) / 4$. The structure is $3 N \mu \mathrm{m}$ wide and $30 \mu \mathrm{m}$ long, including domains with random sizes between 0 and $3 \mu \mathrm{m}$ and random polarization. Each plot corresponds to the average over 100 different realizations of the randomness. (b) The maximum SHI enhancement efficiency as a function of the target direction from the same structure when $N=50$. effect in collinear direction and to the boundary effect in the Cerenkov direction.

\section{CONCLUSIONS}

In summary, we have theoretically analyzed the optimization of SHG by wave-front phase modulation for nonlinear random structures composed of domains with random size and antiparallel polarization. The wave-front phase modulation is modeled by dividing the structure into $N$ segments along the crystal width and applying to them a fundamental wave with variable phases. We have studied the effect of the random structure length and width over SHG under a plane-wave input and found the phase front configurations that lead to an optimized SHG. We have found that the effectiveness in the optimization of the SHI from a nonlinear random structure is affected by the target direction. Our calculations show that, in general, the SHI enhancement efficiency is equal to the one that can be obtained in linear diffusive media. However, in the collinear and the Cerenkov directions, the SHI can have an important contribution from the crystal boundaries. When the disorder in the bulk is present, such contribution can increase the maximum possible enhancement efficiency in the collinear direction, while it decreases that in the Cerenkov direction. When a wave-front phase modulation is included, in the collinear direction the SHI enhancement efficiency is within the limit of the one obtained in linear diffusive media, whereas at the Cerenkov angle, the enhancement efficiency brought by the phase modulation can lead to larger SHI.

\section{ACKNOWLEDGMENTS}

We acknowledge the Ministerio de Economía y Competitividad which supported the work under Grants No. MAT201128665, No. IPT-120000-2010-29, and No. CSD2007-00046. F.J.R. acknowledges support by the Juan de la Cierva program, Grant No. JCI-2009-04860. J.B.A. acknowledges financial support by the Ramon-y-Cajal program, Grant No. RyC-200905489.
[1] M. Baudrier-Raybaut, R. Haïdar, Ph. Kupecek, Ph. Lemasson, and E. Rosencher, Nature (London) 432, 374 (2004).

[2] X. Vidal and J. Martorell, Phys. Rev. Lett. 97, 013902 (2006).

[3] J. Bravo-Abad, X. Vidal, J. L. Domínguez-Juárez, and J. Martorell, Opt. Express 18, 14202 (2010).

[4] F. J. Rodríguez, C. Yao, J. L. Domínguez-Juárez, J. Bravo-Abad, and J. Martorell, Opt. Lett. 36, 1347 (2011).

[5] R. Fischer, S. M. Saltiel, D. N. Neshev, W. Krolikowski, and Yu. S. Kivshar, Appl. Phys. Lett. 89, 191105 (2006).

[6] A. R. Tunyagi, M. Ulex, and K. Betzler, Phys. Rev. Lett. 90, 243901 (2003).

[7] W. Wang, K. Kalinowski, V. Roppo, Y. Sheng, K. Koynov, Y. Kong, C. Cojocaru, J. Trull, R. Vilaseca, and W. Krolikowski, J. Phys. B: At. Mol. Opt. Phys. 43, 215404 (2010).

[8] S. Kawai, T. Ogawa, H. S. Lee, R. C. DeMattei, and R. S. Feigelson, Appl. Phys. Lett. 73, 768 (1998).
[9] N. R. Ivanov, T. R. Volk, L. I. Ivleva, S. P. Chumakova, and A. V. Ginzberg, Crystallogr. Rep. 47, 1023 (2002).

[10] P. Molina, M. O. Ramírez, and L. E. Bausá, Adv. Funct. Mater. 18, 709 (2008).

[11] M. Horowitz, A. Bekker, and B. Fischer, Appl. Phys. Lett. 62, 2619 (1993).

[12] L. Tian, D. A. Scrymgeour, and V. Gopalan, J. Appl. Phys. 97, 114111 (2005).

[13] C. Yao, F. J. Rodríguez, and J. Martorell, Opt. Lett. 37, 1676 (2012).

[14] I. M. Vellekoop and A. P. Mosk, Opt. Lett. 32, 2309 (2007).

[15] A. P. Mosk, A. Lagendijk, G. Lerosey, and M. Fink, Nat. Photon. 6, 283 (2012).

[16] I. M. Vellekoop, A. Lagendijk, and A. P. Mosk, Nat. Photon. 4, 320 (2010). 
[17] S. M. Popoff, G. Lerosey, R. Carminati, M. Fink, A. C. Boccara, and S. Gigan, Phys. Rev. Lett. 104, 100601 (2010).

[18] J. Aulbach, B. Gjonaj, P. M. Johnson, A. P. Mosk, and A. Lagendijk, Phys. Rev. Lett. 106, 103901 (2011).

[19] D. J. McCabe, A. Tajalli, D. R. Austin, P. Bondareff, I. A. Walmsley, S. Gigan, and B. Chatel, Nat. Commun. 2, 447 (2011).

[20] O. Katz, E. Small, Y. Bromberg, and Y. Silberberg, Nat. Photon. 5, 372 (2011).

[21] M. Cui, Opt. Lett. 36, 870 (2011).

[22] D. B. Conkey, A. N. Brown, A. M. Caravaca-Aguirre, and R. Piestun, Opt. Express 20, 1733 (2012).

[23] C. L. Hsieh, Y. Pu, R. Grange, and D. Psaltis, Opt. Express 18, 12283 (2010).

[24] A. D. Yaghjian, Proc. IEEE 68, 248 (1980).

[25] J. D. Jackson, Classical Electrodynamics, 3rd ed. (John Wiley, New York, 1999).

[26] L. Novotny and B. Hetch, Principles of Nano-Optics (Cambridge University Press, New York, 2006).
[27] Ji-Xin Cheng and X. Sunney Xie, J. Opt. Soc. Am. B 19, 1604 (2002).

[28] J. E. Sipe, J. Opt. Soc. Am. B 4, 481 (1987).

[29] K. Sakoda and K. Ohtaka, Phys. Rev. B 54, 5742 (1996).

[30] C. David, A. Tunyagi, K. Betzler, and M. Wöhlecke, Phys. Status Solidi B 6, 2127 (2007).

[31] S. M. Saltiel, Y. Sheng, N. Voloch-Bloch, D. N. Neshev, W. Krolikowski, A. Arie, K. Koynov, and Yu. S. Kivshar, IEEE J. Quantum Electron. 45, 1465 (2009).

[32] W. J. Wang, S. Yan, Y. F. Kong, A. Arie, and W. Krolikowski, Opt. Lett. 35, 3790 (2010).

[33] M. Ayoub, P. Roedig, J. Imbrock, and C. Denz, Opt. Lett. 36, 4371 (2011).

[34] K. Kalinowski, P. Roedig, Y. Sheng, M. Ayoub, J. Imbrock, C. Denz, and W. Krolikowski, Opt. Lett. 37, 1832 (2012).

[35] J. W. Goodman, J. Opt. Soc. Am. 66, 1145 (1976).

[36] I. M. Vellekoop and A. P. Mosk, Opt. Commun. 281, 3071 (2008). 